Supporting Information

\title{
A Strategy Employing a TF-Splinting Duplex Nanoswitch to Achieve Single-Step, Enzyme-Free, Signal-On Detection of L-Tryptophan
}

Xuying D uan ${ }^{+}$, Ziwei Chen ${ }^{+}$, Suming T ang ${ }^{+}$, M eiqiong $\mathrm{G} \mathrm{e}^{+}, \mathrm{H}$ ua W $\mathrm{i}^{+},+$, Y Yifu Guan ${ }^{+}$, and GuojieZhao,+,

+ Department of Biochemistry and M olecular Biology, School of Life Sciences, C hina M edical U niversity, Shenyang, Liaoning 110001, China

${ }^{\ddagger}$ Animal Science and Veterinary M edicine College, Shenyang Agricultural U niversity, Shenyang, Liaoning 110866, China 


\section{Contents}

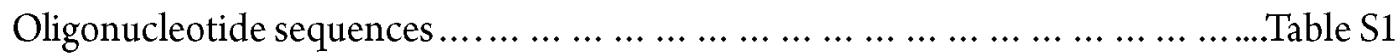

Optimization of nicked duplex length and $\mathrm{MB}$ ...Figure S1

Specificity of the recognition of nicked duplex by TrpR Figure S2

Optimal nick position placement in the structural model . Figure S3

Effect of phosphate groups at nick sites on the TFSD .Figure $S 4$

Comparison of the TFSD between nicked and gapped duplex. Figure S5

Optimization of the ratio of $\mathrm{nON} / \mathrm{MB}$ concentrations... Figure S6

Optimization of the TrpR concentration Figure S7

Binding-switching equilibrium model of the TFSD Figure S8

Optimization of the $\mathrm{pH}$ and $\mathrm{KCl}$ concentration Figure S9

Discrimination of L-Trp and its analogs by the TFSD Figure S10

Effect of bilirubin on TFSD .Figure S11

Fluorescence data obtained from biological samples Figure S12

Cost comparison between the TFSD and ELISA kits Table S2 Assessment of $\operatorname{TrpR}$ purification .Figure $\$ 13$ 


\section{Oligonucleotide sequences}

TableS1 0 ligonucleotides used in experiments.

\begin{tabular}{|c|c|c|}
\hline names & sequences* & characteristics \\
\hline \multirow[t]{2}{*}{ MB-Trp1 } & FAM -5'-AATACACACTGCGAACTAGTTAACTAGTACCTCATGTGTATT-3'- & stem 8 nt, loop $26 \mathrm{nt}$ \\
\hline & Dabcyl & \\
\hline M B-Trp2 & FAM -5'-TACACACTGCGAACTAGTTAACTAGTACCTCATGTGTA-3'-Dabcyl & stem $6 \mathrm{nt}$, loop $26 \mathrm{nt}$ \\
\hline MB-Trp3 & FAM -5'-ACACACTGCGAACTAGTTAACTAGTACCTCATGTGT-3'-Dabcyl & stem $5 \mathrm{nt}$, loop $26 \mathrm{nt}$ \\
\hline MB-Trp4 & FAM -5'-CACACT GCGAACTAGTTAACTAGTACCTCATGTG-3'-Dabcyl & stem 4 nt, loop $26 \mathrm{nt}$ \\
\hline $\operatorname{cON}(18)$ & 5'-GTACTAGTTAACTAGTTC-3' & $18 \mathrm{nt}$ \\
\hline $\operatorname{cON}(20)$ & 5'-GGTACTAGTTAACTAGTTCG-3' & $20 \mathrm{nt}$ \\
\hline $\operatorname{cON}(22)$ & 5'-AGGTACTAGTTAACTAGTTCGC-3' & $22 \mathrm{nt}$ \\
\hline $\operatorname{cON}(24)$ & 5'-GAGGTACTAGTTAACTAGTTCGCA-3' & $24 \mathrm{nt}$ \\
\hline $\operatorname{cON}(26)$ & 5'-TGAGGTACTAGTTAACTAGTTCGCAG-3' & $26 \mathrm{nt}$ \\
\hline $\operatorname{nON}(9 / 9)$ & 5'-GTACTAGTT-3' 5'-AACTAGTTC-3' & left $9 \mathrm{nt}$, right $9 \mathrm{nt}$ \\
\hline $\mathrm{nON}(10 / 10)$ & 5'-GGTACTAGTT-3' 5'-AACTAGTTCG-3' & left $10 \mathrm{nt}$, right $10 \mathrm{nt}$ \\
\hline nON (11/ 11) & 5'-AGGTACTAGTT-3' 5'-AACTAGTTCGC-3' & left $11 \mathrm{nt}$, right $11 \mathrm{nt}$ \\
\hline nON (12/ 12) & 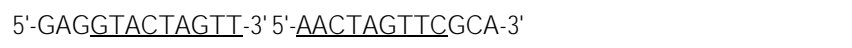 & left $12 \mathrm{nt}$, right $12 \mathrm{nt}$ \\
\hline nON (13/ 13) & 5'-TGAGGTACTAGTT-3' 5'-AACTAGTTCGCAG-3' & left $13 \mathrm{nt}$, right $13 \mathrm{nt}$ \\
\hline cD uplex(competiti & 5'-CTGCGAACTAGTTAACTAGTACCTCA-3' & ON with loop sequence \\
\hline ve duplex) & 3'-GACGCTTGATCAATTGATCATGGAGT-5' & $\operatorname{con}(26)$ \\
\hline rD uplex(random & 5'-CTGCAAACATACGCATACCTGTCTCA-3' & $\mathrm{ON}$ with random sequence \\
\hline duplex) & 3'-GACGTTTGTATGCGTATGGACAGAGT-5' & $\mathrm{CON}$ with random sequence \\
\hline nD uplex(nicked & 5'-CTGCGAACTAGTTAACTAGTACCTCA-3' & $\mathrm{ON}$ with loop sequence \\
\hline duplex) & 3'-GACGCTTGATCAATT-5' 3'-GATCATGGAGT-5' & left 15nt, right 11nt \\
\hline $\mathrm{nON}(10 / 12)$ & 5'-AGGTACTAGT-3' 5'-TAACTAGTTCGC-3' & left $10 \mathrm{nt}$, right $12 \mathrm{nt}$ \\
\hline $\operatorname{nON}(9 / 13)$ & 5'-AGGTACTAG-3' 5'-ITAACTAGTTCGC-3' & left $9 \mathrm{nt}$, right $13 \mathrm{nt}$ \\
\hline $\mathrm{nON}(8 / 14)$ & 5'-AGGTACTA-3' 5'-GTTAACTAGTTCGC-3' & left $8 \mathrm{nt}$, right $14 \mathrm{nt}$ \\
\hline $\mathrm{nON}(7 / 15)$ & 5'-AGGTACT-3' 5'-AGTTAACTAGTTCGC-3' & left $7 \mathrm{nt}$, right $15 \mathrm{nt}$ \\
\hline $\operatorname{nON}(6 / 16)$ & 5'-AGGTAC-3' 5'-TAGTTAACTAGTTCGC-3' & left $6 \mathrm{nt}$, right $16 \mathrm{nt}$ \\
\hline $\mathrm{nON}(12 / 10)$ & 5'-AGGTACTAGTTA-3' 5'-ACTAGTTCGC-3' & left $12 \mathrm{nt}$, right $10 \mathrm{nt}$ \\
\hline $\operatorname{nON}(13 / 9)$ & 5'-AGGTACTAGTTAA-3' 5'-CTAGTTCGC-3' & left $13 \mathrm{nt}$, right $9 \mathrm{nt}$ \\
\hline $\mathrm{nON}(14 / 8)$ & 5'-AGGTACTAGTTAAC-3' 5'-IAGTTCGC-3' & left $14 \mathrm{nt}$, right $8 \mathrm{nt}$ \\
\hline $\mathrm{nON}(15 / 7)$ & 5'-AGGTACTAGTTAACT-3' 5'-AGTTCGC-3' & left $15 \mathrm{nt}$, right $7 \mathrm{nt}$ \\
\hline $\operatorname{nON}(16 / 6)$ & 5'-AGGTACTAGTTAACTA-3' 5'-GTTCGC-3' & left $16 \mathrm{nt}$, right $6 \mathrm{nt}$ \\
\hline nON (11/ 11p) & 5'-AGGTACTAGTT-3' 5'-p-AACTAGTTCGC-3' & $\begin{array}{l}\text { left } 11 \mathrm{nt} \text {, right } 11 \mathrm{nt} \text { (5' } \\
\text { phosphorylated) }\end{array}$ \\
\hline nON (10/12p) & 5'-AGGTACTAGT-3' 5'-p-IAACTAGTTCGC-3' & $\begin{array}{l}\text { left } 10 \mathrm{nt} \text {, right } 12 \mathrm{nt} \\
\text { phosphorylated) }\end{array}$ \\
\hline nON (9/13p) & 5'-AGGTACTAG-3' 5'-p-ITAACTAGTTCGC-3' & $\begin{array}{l}\text { left } 9 \mathrm{nt} \text {, right } 13 \mathrm{nt} \quad 5^{\prime} \\
\text { phosphorylated) }\end{array}$ \\
\hline gON 1 & 5'-AGGTACTAGT-3' 5'-AACTAGTTCGC-3' & left $10 \mathrm{nt}$, right $11 \mathrm{nt}$ \\
\hline
\end{tabular}


*T he recognition sequences of $T r p R$ are underlined; the stems of the $M B$ are wave-lined.

\section{Optimization of nicked duplex length and $M B$}

We used two short oligonucleotides ( $\mathrm{nO} \mathrm{N}$ s) to form nicked duplex as the unstacked conformation, and used single long oligonucleotides ( $\mathrm{CON}$ ) to form continuous duplex to simulate the ideal stacked conformation. T he nO N s were the nicked counterparts of cO N s, for example, the nicked counterpart of the $22 \mathrm{nt}$ long cO N (22) was two short $11 \mathrm{nt}$ long $n O N(11 / 11)$. The optimal length of loop duplex and MB should present the best fluorescence response of structural switching.

The M B we used contained a $26 \mathrm{nt}$ long loop. In the middle of the loop, an $18 \mathrm{nt}$ long recognition sequence of T rpR was embedded. We designed duplex length ranging from $18 \mathrm{bp}$ (recognition sequence) to $26 \mathrm{bp}$ (the whole loop). When the length was too short, such as formed by $\mathrm{nON}(9 / 9)$, the stacked conformation formed by $\mathrm{CON}(18)$ only slightly increased the fluorescence. When the length was too long, such as formed by nON (12/12) or nON (13/ 13), nON itself without splinting was able to open the MB. We found the best fluorescence change occurred between $\mathrm{nON}(11 / 11)$ and $\mathrm{CON}(22)$, which represented the optimal length of the nicked duplex. (Figure S1A E)

We also tested four M Bs ( M B-T rpR 1 4) with different stem lengths. We compared their fluorescence difference between $\mathrm{CON}$ and $\mathrm{nON}$ groups. Results showed that M B-T rpR2 provided the best fluorescence change. (FigureS1F $\sim$ ) 
A

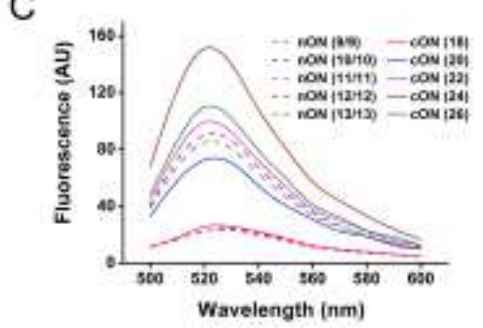

$\mathrm{F}$

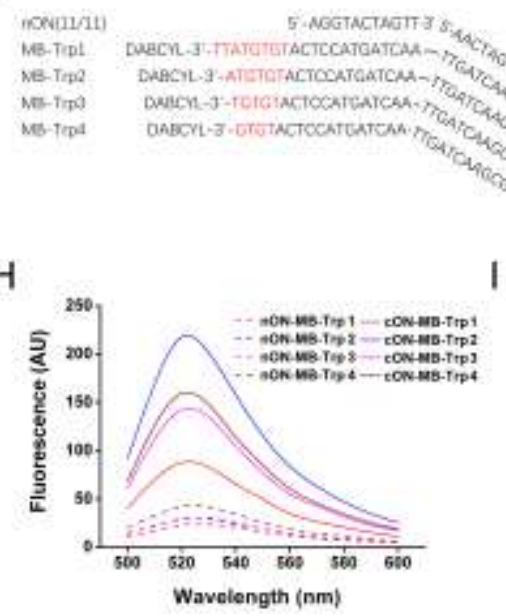

B

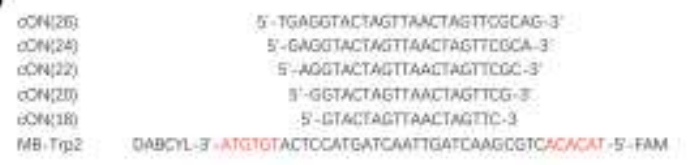

$E$

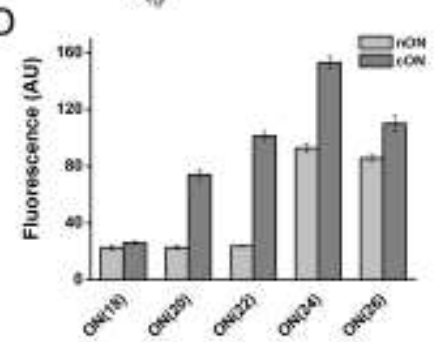

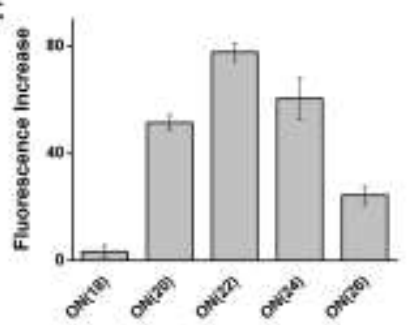

G

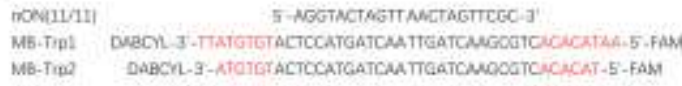

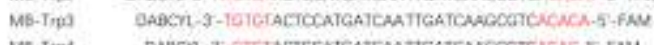

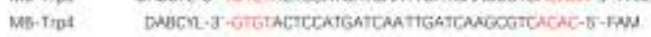

Figure S1. The optimal duplex length and MB for monitoring conformational change. (A) Schematic of nON S hybridized with M B. Red represents stem part of M B. (B) Schematic of $\mathrm{CON}$ s hybridized with M B. (C) Fluorescence as a function of wavelength affected by different $\mathrm{nON} / \mathrm{CON}$ lengths. 'cON' represents ' $\mathrm{MB}+\mathrm{cON}$ '. 'nON' represents ' $M B+n O N$ '. (D) Fluorescence intensity $(\lambda=520 \mathrm{~nm})$ of $(C)$. (E) Fluorescence increase of $c O N$ in comparison to nO N, calculated from panel (D) . (F) Schematic of nO N s hybridized with M Bs. Red represents stem part of M B. (G) Schematic of $\mathrm{CO}$ N s hybridized with M B. (H) Fluorescence as a function of wavelength affected by different M Bs. (I) Fluorescence intensity $(\lambda=520 \mathrm{~nm})$ of $(H)$. 'cON' represents ' $\mathrm{MB}+\mathrm{cON}$ '. 'nON' represents 'MB+nON'. (J) Fluorescence increase of $\mathrm{CON}$ in comparison to $\mathrm{nON}$, calculated from panel (I). D ata points are presented as mean \pm $\operatorname{SD}(n=3)$.

\section{Specificity of the recognition of nicked duplex by $\operatorname{TrpR}$}


A

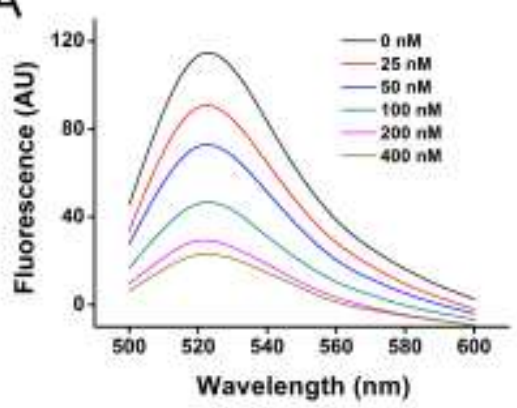

C

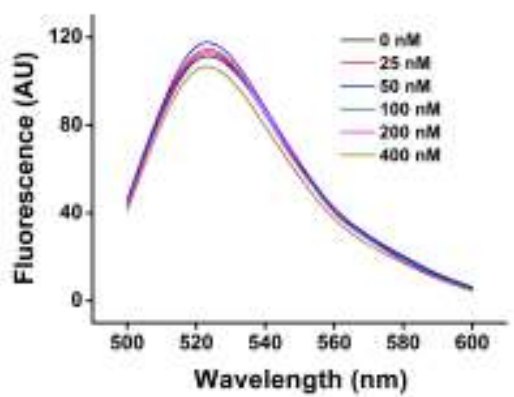

E

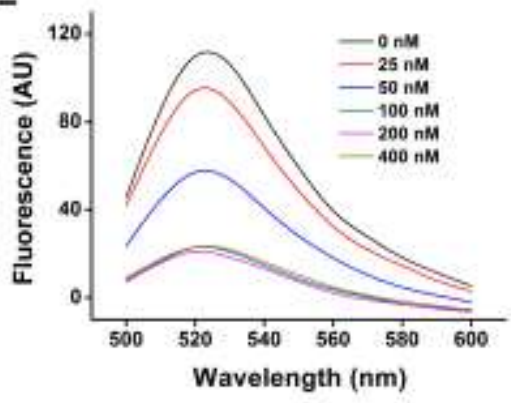

B

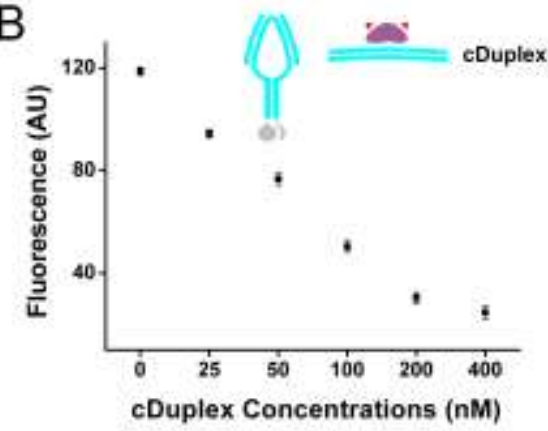

D

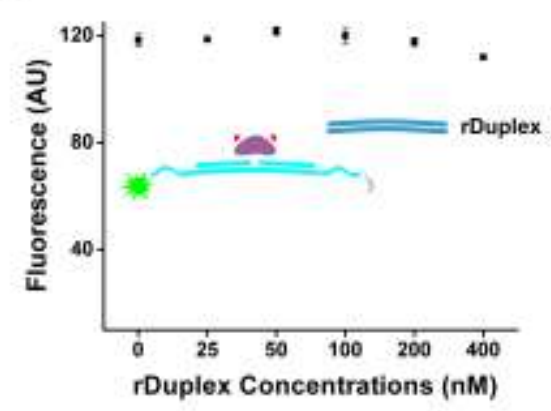

$\mathrm{F}$

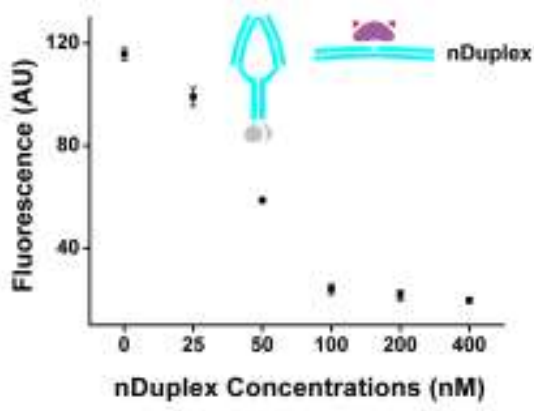

Figre $\Omega$ Sequence-specific recognition of the nicked duplex by TrpR. (A) Competitive inhibition of TFSD by CD uplex of increased concentration from 0 to $400 \mathrm{nM}$. (B) Fluorescence intensity $(\lambda=520 \mathrm{~nm})$ of (A). (C) Competitive inhibition of TFSD by rD uplex of increased concentration from 0 to $400 \mathrm{nM}$. (D) Fluorescence intensity $(\lambda=520 \mathrm{~nm})$ of (C). (E) Competitive inhibition of TFSD by nD uplex of increased concentration from 0 to $400 \mathrm{nM}$. (F) Fluorescence intensity ( $\lambda=520 \mathrm{~nm}$ ) of (E). $50 \mathrm{nM} \mathrm{M} \mathrm{B-Trp2} \mathrm{and} 100 \mathrm{nM} \mathrm{nON} \mathrm{(9/13)} \mathrm{were} \mathrm{used.} \mathrm{Datapoints} \mathrm{are}$ presented as mean $\pm S D(n=3)$.

\section{Optimal nick position placement in the structural model}

TrpR has been reported to be a homodimer protein. ${ }^{1}$ Each monomer has six helices (helices A-F). Among them, $\mathrm{D}$ and $\mathrm{E}$ helices form a DNA reading head. The binding of L-Trp tightens apo-repressor into holorepressor with a well-formed DNA-reading head, which is suitable to be fitted into two consecutive major grooves of the recognition sequence. ${ }^{1}$ As such, TrpR bind a dyad-symmetry structure, characterized by two 'GNACTAG' spaced by four base pairs. ${ }^{2}$ The conservative 'GNACTAG' sequence constitutes the major grooves for binding.

According to the tertiary structure of TrpR-duplex, nick site of nON(11/11) is closer to TrpR than that of the optimal nON(9/13) is. Our results in Figure 2 suggest that the nick positions on the side of duplex 
far from TrpR behave relatively better for TFSD structure-switching than that on the side facing TrpR.

(Figure S3)

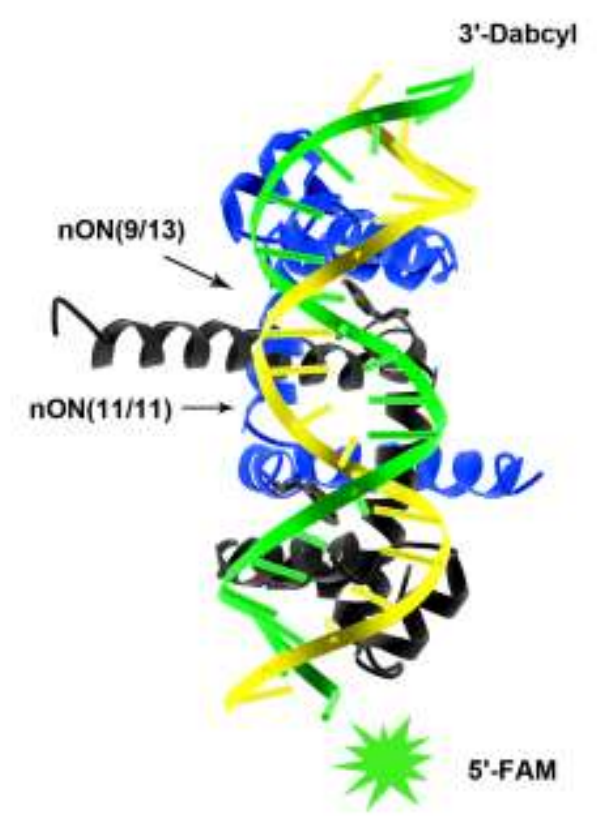

FigureS3. 0 ptimal nick position on the tertiary structure of T rpR-duplex complex. (1TRO) Blue and black represent protein dimers. G reen and yellow represent bound D N A duplex. G reen strand represents M B labeled with 5' FAM and 3' Dabcyl. Yellow strand represents cON or nON. The nick positions of nON (9/13) and nON (11/11) in yellow strand are indicated by arrows.

Effect of phosphate groups at nick sites on the TFSD
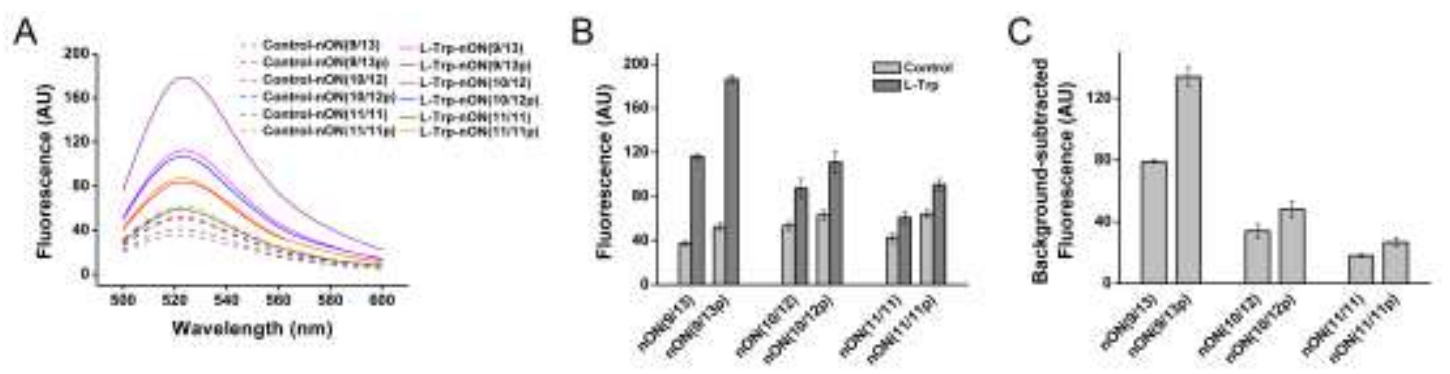

Figure S4. Effect of addition of phosphate groups at duplex nick sites on L-Trp detection using TFSD. (A) Fluorescence intensity $(\lambda=520 \mathrm{~nm})$ affected by phosphate group, with and without L-T $\mathrm{r}$. 'Control' and 'L-Trp' are defined as in Figure 2. Samples nON (9/13p), nON (10/12p) and nON (11/11p) are the phosphorylated forms of $n O N(9 / 13), n O N(10 / 12)$ and $n O N(11 / 11)$, respectively. (B) Fluorescence affected by phosphate groups in response to L-T rp, calculated from panel (A). The sequences of M B ( M B-T rp2) and $\mathrm{nON}$ s are listed in T able S1. D ata points are presented as mean $\pm S D(n=3)$. 


\section{Comparison of the TFSD between nicked and gapped duplex}
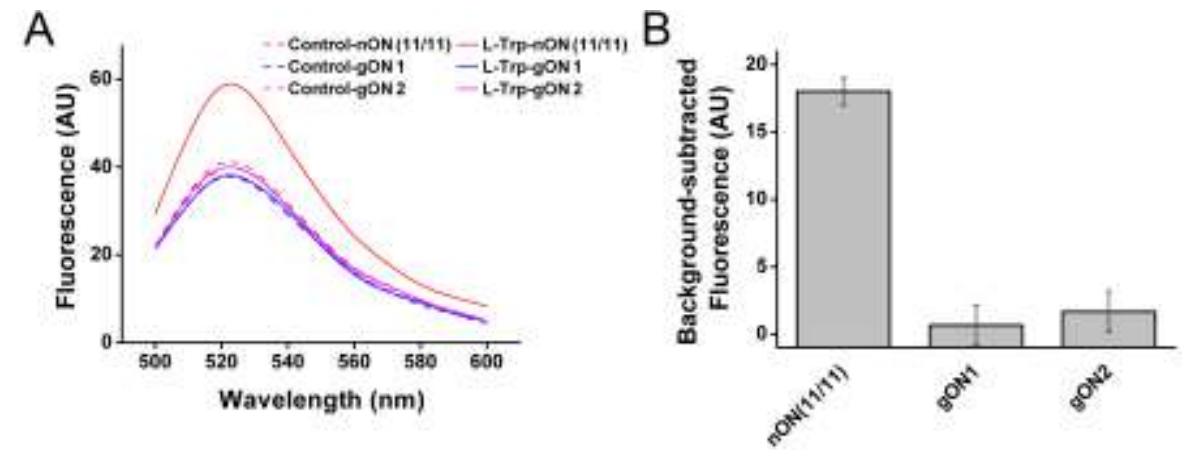

Figure 55. Gap instead of nick failing to open the M B using TFSD. (A) Fluorescence as a function of wavelength affected by gaps in duplex, with and without L-T rp. 'Control' represents ' $\mathrm{MB}+\mathrm{nON} / \mathrm{gON}+\mathrm{TrpR}$ ' as background. 'LTrp' represents ' $M B+n O N / g O N+T r p R+L-T r p$ '. (B) Fluorescence increase $(\lambda=520 \mathrm{~nm})$ affected by gaps in duplex, in response to $L-T r p$, calculated from panel $(A)$. Data points are presented as mean $\pm S D(n=3)$.

\section{Optimization of the ratio of $\mathrm{nON} / \mathrm{MB}$ concentrations}
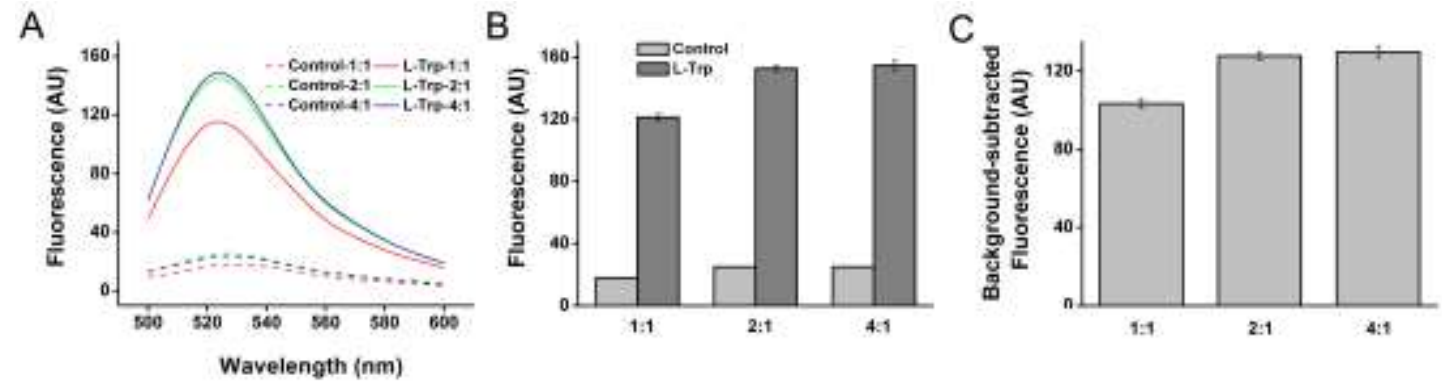

Figure S6. Effect of different ratios of $\mathrm{nON}$ / M B concentrations on L-T rp detection using T FSD. (A) Fluorescence intensity $(\lambda=520 \mathrm{~nm})$ using either $50,100,200 \mathrm{nM} \mathrm{nON}$ s versus $50 \mathrm{nM} \mathrm{MB},(1: 1,2: 1$ and $4: 1$, respectively), with and without L-Trp. 'Control' and 'L-Trp' are defined as in Figure 2. (B) Fluorescence increase versus oligonucleotide concentration ratios, in response to $L-T r p$, calculated from panel (A). Data points are presented as mean $\pm S D(n=3)$.

\section{Optimization of the TrpR concentration}



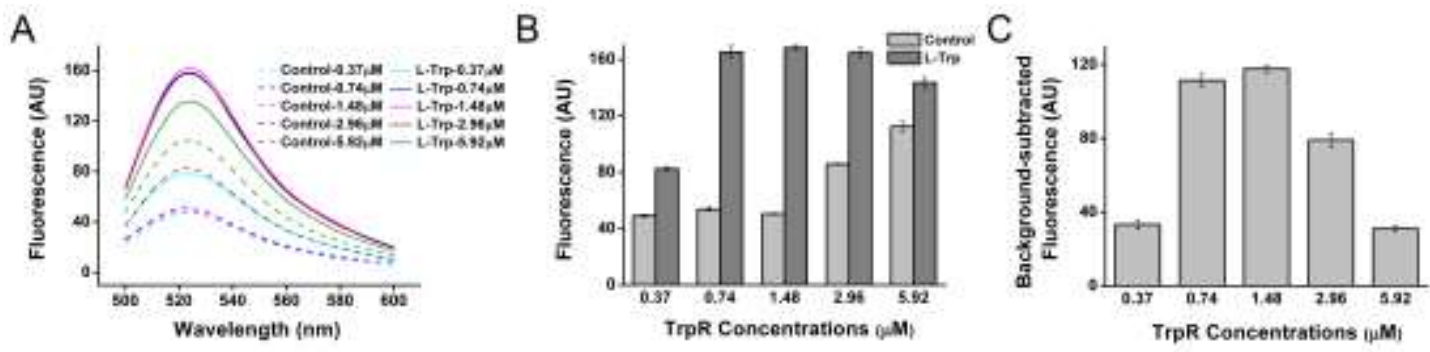

FigreS7. Effect of TrpR concentration on L-T rp detection using TFSD. (A) Fluorescence intensity $(\lambda=520 \mathrm{~nm})$ as a function of TrpR concentration, from $0.37 \mu \mathrm{M}$ to $5.92 \mu \mathrm{M}$, with and without $100 \mu \mathrm{M} \mathrm{L-Trp.} \mathrm{'Control'} \mathrm{and} \mathrm{'L-Trp'}$ are defined as in Figure 2. (B) Fluorescence increase as a function of TrpR concentration, in response to L-Trp, calculated from panel (A). D ata points are presented as mean $\pm S D(n=3)$.

\section{Binding-switching equilibrium model of the TFSD}

According to TFSD principle, the whole system contained two binding equilibriums (Kd of tryptophanTrpR interaction and $K d$ of TrpR-duplex interaction) and the switching equilibrium (Ks of MB) as well. We used a simplified equilibrium model of TFSD focusing on $K d$ of TrpR-duplex interaction $(K d=k 1 / k 2)$ and Ks $(K s=k 3 / k 4)$. (Figure 88$)$

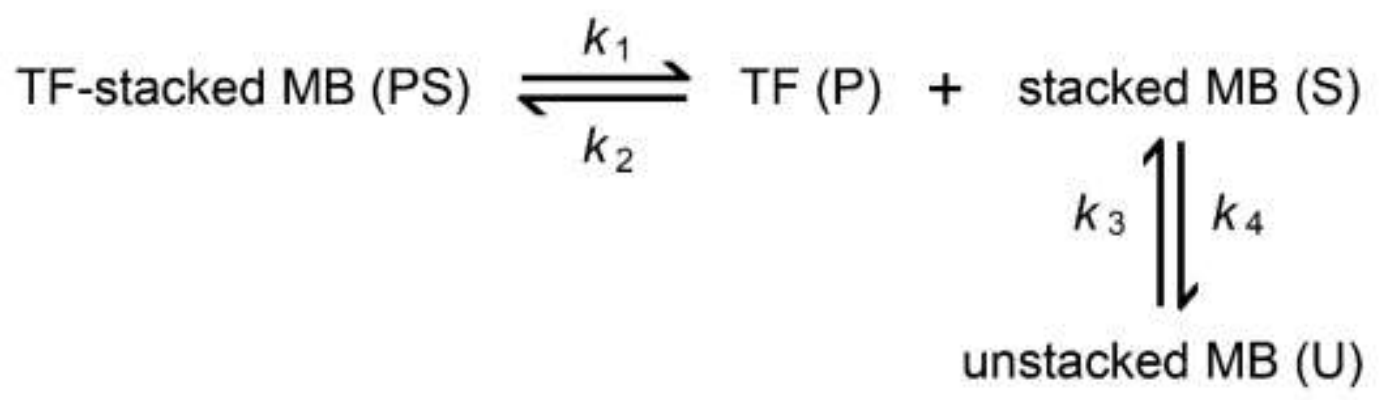

FigureS8. Reaction equilibrium model in TFSD. 'PS' represents ‘TF-stacked MB'. 'P' represents 'TF'. 'S' represents 'stacked MB'. 'U' represents 'unstacked MB'.

At steady state

$$
k 1[\mathrm{PS}]=k 2[\mathrm{P}][\mathrm{S}]
$$

Then

$$
[\mathrm{S}]=\frac{k 1[\mathrm{PS}]}{k 2[\mathrm{P}]}
$$

At steady state

$$
k 4[\mathrm{~S}]=k 3[\mathrm{U}]
$$

Then

$$
[\mathrm{U}]=\frac{k 4}{k 3}[\mathrm{~S}]=\frac{k 4}{k 3} \frac{k 1}{k 2} \frac{[\mathrm{PS}]}{[\mathrm{P}]}
$$

The detectable saturation fraction is defined as apparent $\mathrm{Y}$ 


$$
\text { apparent } Y=\frac{[\text { MBopen }]}{[\text { MBtotal }]}=\frac{[\mathrm{PS}]+[\mathrm{S}]}{[\text { MBtotal }]}
$$

Since $[S]$ is trace and ignorable because of free MB closing

$$
\text { apparent } Y \approx \frac{[\mathrm{PS}]}{[\text { MBtotal }]}=\frac{[\mathrm{PS}]}{[\mathrm{PS}]+[\mathrm{S}]+[\mathrm{U}]}
$$

Substitute (1) for [S], and (2) for [U]

$$
\text { apparent } \mathrm{Y}=\frac{[\mathrm{P}]}{[\mathrm{P}]+\frac{k 1}{k 2}\left(1+\frac{k 4}{k 3}\right)}
$$

Given

$$
K \mathrm{~d}=\frac{k 1}{k 2} \quad K \mathrm{~s}=\frac{k 3}{k 4}
$$

Then

$$
\text { apparent } \mathrm{Y}=\frac{[\mathrm{P}]}{[\mathrm{P}]+K \mathrm{~d}\left(1+\frac{1}{K S}\right)}
$$

Define

$$
\text { apparent } K \mathrm{~d}=K \mathrm{~d}\left(1+\frac{1}{K S}\right)
$$

Since

$$
K s \ll 1
$$

Therefore

$$
\text { apparent } K \mathrm{~d} \gg K \mathrm{~d}
$$

Since $K s$ must favor the nonbinding state $(K s<<1$ for $\mathrm{MB})$ to provide clear background before binding and switching occur, the TF-duplex affinity could be decreased by Ks, resulting in an increased apparent Kd. ${ }^{3}$ This is consistent with the results in Figure S7.

\section{Optimization of the $\mathrm{pH}$ and $\mathrm{KCl}$ concentration}

We optimized the $\mathrm{pH}$ and ion concentration for the assay. With the increase of $\mathrm{pH}$ value from 7.0-8.5, the fluorescence increment triggered by tryptophan increased obviously. Condition at $\mathrm{pH} 8.5$ gave the best signal response and were used in the following experiments. (Figure S9A C)

In terms of ions, we found that $\mathrm{KCl}$ had better effects on TFSD than $\mathrm{NaCl}$ did. Results also showed that high $\mathrm{KCl}$ concentration increased fluorescence response triggered by L-Trp, and $100 \mathrm{mM} \mathrm{KCl}$ produced the best signal response of TFSD. (Figure S9D F) 
A

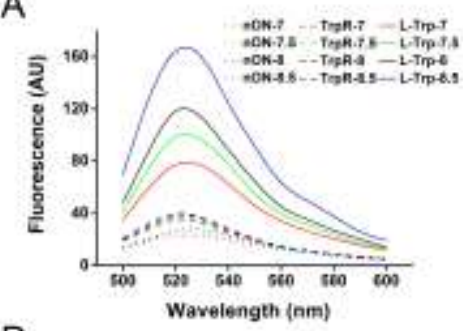

D

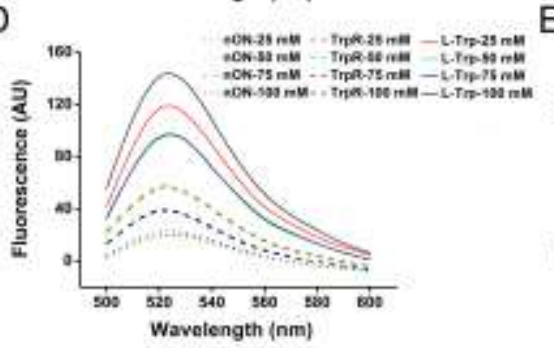

B

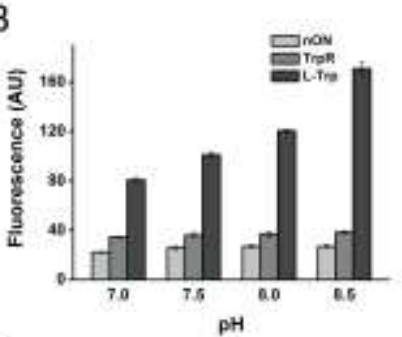

E

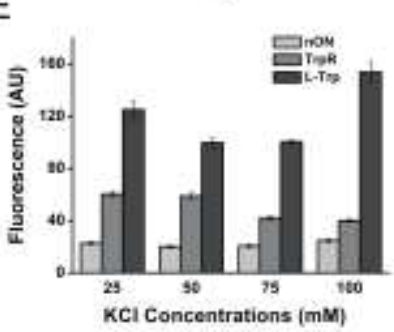

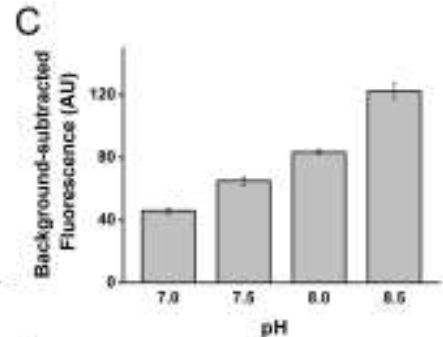

$\mathrm{F}$

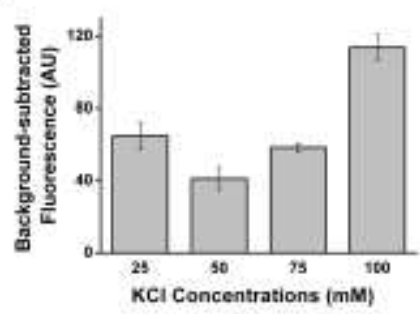

Figures9. Effects of pH and $\mathrm{KCl}$ concentration on L-Trp detection using TFSD. (A) Fluorescence as a function of wavelength for $\mathrm{pH}$ ranging from 7.0 to 8.5. 'nON' represents ' $\mathrm{MB}+\mathrm{nON}$ '. 'TrpR' represents ' $\mathrm{MB}+\mathrm{nON}+\mathrm{TrpR}$ ' as background. 'L-Trp' represents 'MB+nON+TrpR+L-Trp'. (B) Fluorescence intensity $(\lambda=520 \mathrm{~nm})$ as a function of $\mathrm{pH}$, obtained from panel (A). (C) Fluorescence increase as a function of $\mathrm{pH}$, in response to L-Trp, calculated from panel (B). (D) Fluorescence as a function of wavelength for $\mathrm{KCl}$ concentrations ranging from $25 \mathrm{mM}$ to $100 \mathrm{mM}$. 'nON', 'TrpR' and 'L-Trp' are defined as in panel (A). (E) Fluorescence intensity $(\lambda=520 \mathrm{~nm})$ as a function of $\mathrm{KCl}$ concentration, obtained from panel (D) . ( F) Fluorescence increase as a function of $\mathrm{KCl}$ concentration, in response to $L-T r p$, calculated from panel (E). Data points are presented as mean $\pm S D(n=3)$.

\section{Discrimination of $L-T r p$ and its analogs by the TFSD}

A

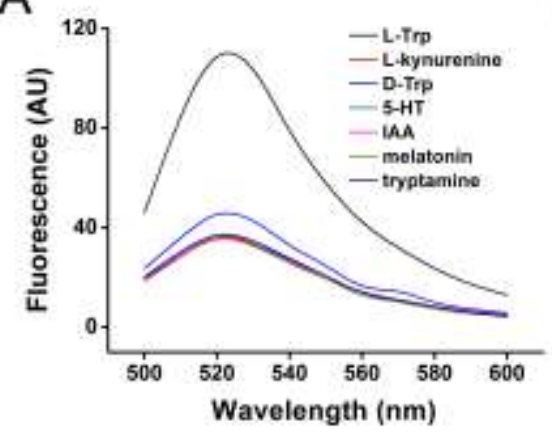

B

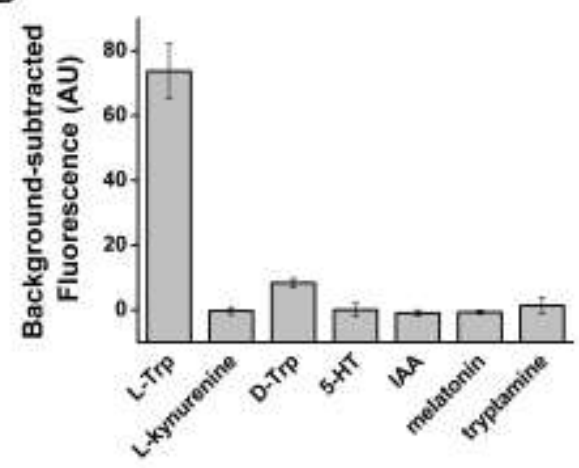

FigureS10. Discrimination of L-T rp from its analogs using TFSD. (A) Fluorescence as a function of wavelength, in response to $10 \mu \mathrm{M} \mathrm{L-Trp}$ and its six analogs ( $100 \mu \mathrm{M}$ each). 5-HT represents 5-hydroxytryptamine; IAA represents indoleacetic acid. (B) Background-subtracted fluorescence intensity $(\lambda=520 \mathrm{~nm})$, calculated from panel (A). Data points are presented as mean $\pm S D(n=3)$.

\section{Effect of bilirubin on TFSD}


We measured fluorescence of bilirubin ( $0-5 \mu \mathrm{M})$ in TFSD buffer in our experimental conditions. Results showed that its fluorescence at this concentration was very close to the background. (Figure S11A, B) The bilirubin with the same concentrations was spiked into the TFSD system. Results showed that tryptophan-induced signals at 0-5 $\mu \mathrm{M}$ bilirubin were similar. (Figure S11C, D, E)

Significantly, the fluorescence of $0.05 \mu \mathrm{M}$ FAM (i.e. MB's concentration) in Figure S11C was much higher (either closed or opened states of M B) than that of $5 \mu \mathrm{M}$ bilirubin in Figure S11A. This may be due to the quantum yields of FAM (as an excellent commercial fluorescent dye) are much higher than that of bilirubin, though they have similar fluorescent spectrum. Therefore, the fluorescence interference by bilirubin at this concentration was ignorable.

Regarding serum detection experiments, $10 \mu \mathrm{L}$ serum was added into a final $100 \mu \mathrm{L}$ TFSD buffer. According to Figure S11, even $50 \mu \mathrm{M}$ bilirubin in serum ( $5 \mu \mathrm{M}$ in final TFSD buffer) did not make an obvious interference with detection. In addition, the normal concentration of bilirubin in human serum is less than $17.1 \mu \mathrm{M}(<50 \mu \mathrm{M})$, which can hardly affect TFSD.

A

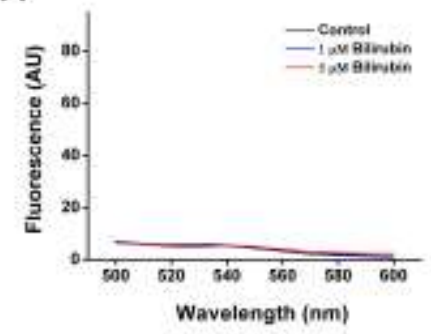

B

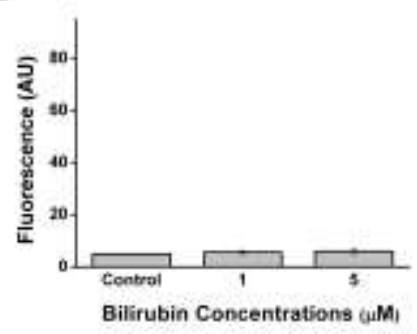

C
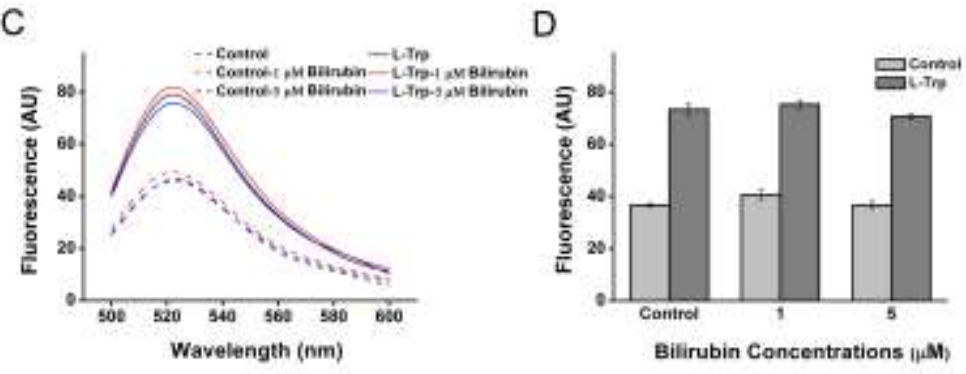

E

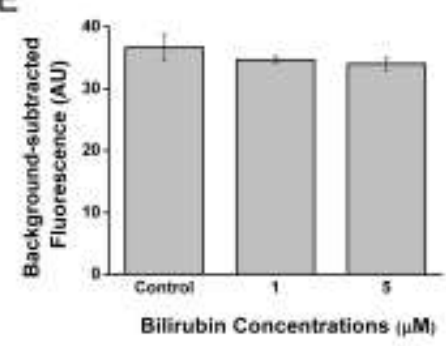

Figures]1 Effects of bilirubin on fluorescence in TFSD. (A) Fluorescence as a function of wavelength for $0,1,5 \mu \mathrm{M}$ bilirubin in TFSD buffer. (B) Fluorescence intensity $(\lambda=520 \mathrm{~nm}$ ) at different bilirubin concentrationsfrom panel (A). (C) Fluorescence as a function of wavelength for detection of $4 \mu \mathrm{M}$ L-Trp by TFSD with 0,1,5 $\mu \mathrm{M}$ bilirubin. 'C ontrol' represents ' $\mathrm{MB}+\mathrm{nON}+\mathrm{TrpR}$ ' as background. 'L-Trp' represents ' $\mathrm{MB}+\mathrm{nON}+\mathrm{Trp} R+L-T r p$ '. (D) Fluorescence intensity $(\lambda=520 \mathrm{~nm})$ at different bilirubin concentrations from panel $(C)$. (E) Fluorescence increase at different bilirubin concentrations, in response to L-Trp, calculated from panel (D). Data points are presented as mean $\pm S D$ ( $n$ $=3$ ).

\section{Fluorescence data obtained from biological samples}


A

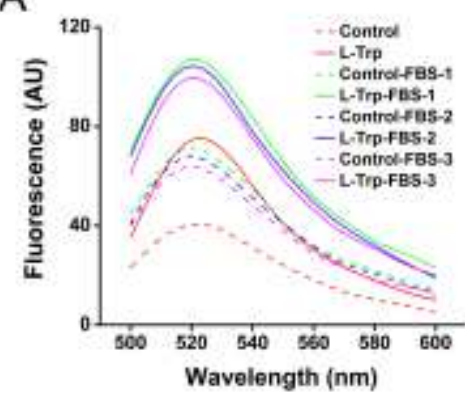

B

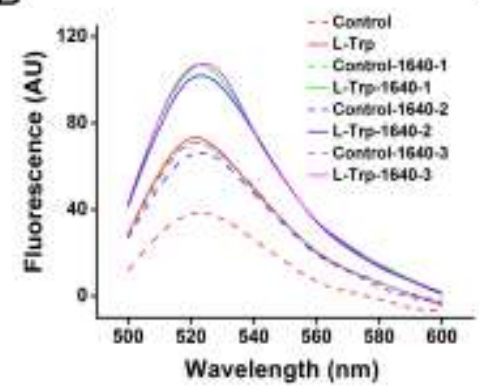

C

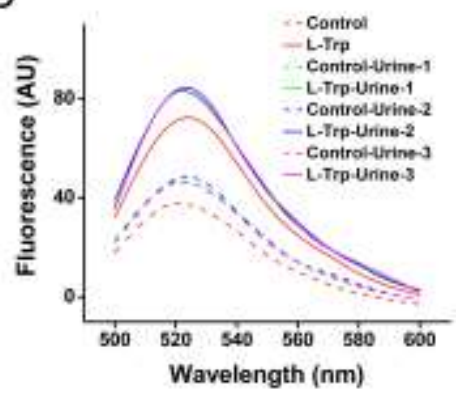

FigreS12. F luorescence as a function of wavelength for three biological samples spiked or unspiked with L-Trp. (A) FBS samples spiked or unspiked with $40 \mu \mathrm{M}$ L-T rp. 'Control' represents unspiked standard sample. 'L-Trp' represents spiked standard sample. 'Control-FBS' represents unspiked FBS. 'L-Trp-FBS' represents spiked FBS. (B) 1640 medium samples spiked or unspiked with $40 \mu \mathrm{M}$ L-Trp. 'Control-1640' represents unspiked 1640 medium. 'L-Trp1640 ' represents spiked 1640 medium. (C) U rine samples spiked or unspiked with $40 \mu \mathrm{M}$ L-Trp. 'Control-Urine' represents unspiked Urine. 'L-Trp-Urine' represents spiked Urine.

\section{Cost comparison between the TFSD and ELISA kits}

We calculated the cost of TFSD in detail, and compared our method with the commonly used commercial ELISA kit. (Table S2)

TableS2 M aterials and cost comparison between the TFSD and commercial tryptophan ELISA kit.

\begin{tabular}{lcc}
\hline main reagents & TFSD nanoswitch & competitive EL ISA kit \\
\hline proteins & TrpR & antiserum, anti-I gG , peroxidase \\
labelling & labelling oligos with fluorophore & labelling anti-IgG with peroxidase \\
oligos & oligos & -- \\
substrates & -- & TM B \\
other reagents & -- & derivatization reagent, precipitating \\
& & reagent, etc. \\
\hline cost & $\$ 0.16 /$ sample* & $\$ 7.3-\$ 14.6 /$ sample $\$ 700-\$ 1400 / 96$ \\
\end{tabular}

*Calculated from materials used in experiments.

In comparison to ELISA, though fluorescence-labeled oligos are required in TFSD method, only one protein material ( $\operatorname{TrpR}$ ) is required, and no substrate is required. The production and labelling of oligonucleotides are relatively easier and cheaper than that of proteins. The material cost in TFSD has been calculated to be relatively cheaper than the common ELISA commercial kit price. (Table S2)

\section{Assessment of TrpR purification}




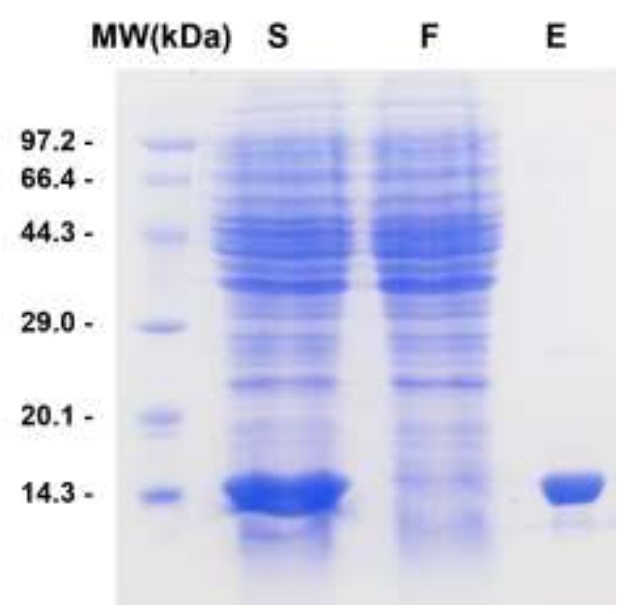

Figure S13. SDS-PAGE assessment of TrpR purification by N i-NTA affinity chromatography. Lane $S$ is the supernatant of the lysed cells after induction and centrifugation. Lane $F$ is the flow-through after beads binding. Lane E is the eluted TrpR.

\section{REFERENCES}

1. Zhao, D.; Arrowsmith, C. H.; Jia, X.; Jardetzky, O. Refined solution structures of the Escherichia coli trp holo- and aporepressor. J. Mol. Biol. 1993, 229 (3), 735-46.

2. Reedstrom, R. J.; Brown, M. P.; Grillo, A.; Roen, D.; Royer, C. A. Affinity and specificity of trp repressor-DNA interactions studied with fluorescent oligonucleotides. J.Mol. Biol. 1997, 273 (3), 572-85.

3. Vallee-Belisle, A.; Plaxco, K. W. Structure-switching biosensors: inspired by Nature. Curr. Opin. Struct. Biol. 2010, 20 (4), 518-26. 